\title{
A Revised Criterion for the Portevin-Le Châtelier Effect Based on the Strain-Rate Sensitivity of the Work-Hardening Rate
}

\begin{abstract}
PETER VAN LIEMPT and JILT SIETSMA
An improved analysis is presented of the stability of plastic deformation under conditions where dynamic strain aging (DSA) occurs, which leads to instabilities known as the Portevin-Le Châtelier (PLC) effect. It is shown that PLC instabilities can occur for conditions that are not covered by the currently prevailing criterion presented by Estrin and Kubin (1991), which focuses on a negative strain rate sensitivity of the flow stress, caused by interactions of solutes with thermally activated glide of mobile dislocations. The current analysis recognizes that the strain-rate sensitivity of the flow stress consists of two contributions, one associated with glide of mobile dislocations and the second with work hardening, related to storage of immobile dislocations. In this paper, an instability criterion is proposed that takes into account the possibility of a negative strain-rate sensitivity of the work-hardening rate, which is caused by diffusion of solutes to immobile dislocations. The latter contribution leads to an extended instability criterion. This criterion also provides an explanation for the existence of a critical strain above which instabilities occur. In this article, previously published tensile test data are used to show that a negative strain-rate sensitivity of the work-hardening rate, which influences significantly the occurrence of the PLC effect, can indeed occur under DSA conditions.
\end{abstract}

DOI: $10.1007 / \mathrm{s} 11661-011-0850-5$

(C) The Author(s) 2011. This article is published with open access at Springerlink.com

\section{INTRODUCTION}

THE dynamic strain aging (DSA) phenomenon, which is also commonly referred to as the Portevin-Le Châtelier (PLC) effect, is an anomaly of the mechanical properties of many metals and alloys. DSA manifests itself in the form of instabilities in the flow-stress curve during plastic deformation in a certain range of temperatures and strain rates. The instabilities are associated with repetitive stationary or propagating bands of localized deformation. Physically, DSA is related to static strain aging, which occurs after deformation, and it is the cause of yield phenomena (Lüders effect). Lüders instabilities are also propagating deformation bands, but in contrast to PLC instabilities, they are not repetitive.

In industrial processes like deep drawing, both types of instability are the cause of surface defects and are therefore undesirable. The Lüders phenomenon can be suppressed by temper rolling prior to the sheet-forming

PETER VAN LIEMPT, Senior Researcher, is with the Strip Metallurgy for Automotive and Packaging, Tata Steel Research, Development \& Technology, Postbus 10000 (3G37), 1970 CA IJmuiden, Netherlands, and is also a Ph.D. Researcher with the Werktuigbouwkunde, Maritieme Techniek en Technische Materiaalkunde, Materials Science and Engineering, Microstructure Control in Metals, Technische Universiteit Delft, Kamer : 8D-1-08 Gebouw 34, Mekelweg 2, 2628 CD Delft, The Netherlands. Contact e-mails: p.vanliempt@tudelft.nl and Peter.van-Liempt@TATASteel.com JILT SIETSMA, Professor, is with the Werktuigbouwkunde, Maritieme Techniek en Technische Materiaalkunde, Materials Science and Engineering, Microstructure Control in Metals, Technische Universiteit Delft.

Manuscript submitted June 2, 2010.

Article published online October 13, 2011 process. For instabilities caused by DSA, an easily applicable countermeasure is not readily available because DSA occurs when aging conditions are met during deformation and can only be avoided by a careful choice of the temperature and strain rate for the sheet-forming process. A reliable instability criterion is an important prerequisite for making that choice.

The prevailing criterion, proposed by Estrin and Kubin recognizes that DSA is caused by the diffusion of solutes to dislocations. ${ }^{[1-4]}$ The dislocations eventually become locked by the acquired solute atmosphere, which affects the flow stress, in particular its dependence on strain rate and temperature. Commonly, PLC stabilities caused by DSA are explained by assuming that the strain-rate sensitivity of the flow stress becomes negative because of dislocation locking.

Negative strain-rate sensitivity is accepted generally as a cause of plastic instability, but the effect of negative strain rate on the work-hardening rate has so far not been analyzed in the literature. In the current paper, we analyze the general case in which both the flow stress and the work-hardening rate are functions of strain rate. This results in a new formulation of the criterion for flow instability, in which the strain-rate sensitivity of both the flow stress and the work-hardening rate are taken into account. Its validity will be discussed based on previously published experimental data.

\section{BACKGROUND}

In the literature, modeling of the dynamic features of the PLC effect (stress drops and PLC-band nucleation and propagation) has received wide attention. Physically, 
the PLC effect is explained commonly ${ }^{[1,2,5]}$ by a dynamic interaction between mobile dislocations and diffusing solutes. Mobile dislocations that are arrested temporarily at local obstacles (which is the origin of the thermal flow-stress contribution) will become pinned by solute atmospheres that form while the dislocation is immobilized. This causes an increase of the thermal flow-stress contribution. At low strain rates, the waiting time increases; consequently the pinning becomes more prominent and the flow stress increases. At high rates, the diffusion rate of the solutes is too low to cause pinning, and the thermal flow-stress contribution is then an increasing function of the strain rate, as is normal for thermally activated dislocation propagation. In most of these models, the hypothesis of negative strain-rate sensitivity is the central element. This concept was introduced by Penning ${ }^{[3]}$ based on the assumption that the constitutive equation can be separated in the variables $\varepsilon$ and $\dot{\varepsilon}$, where $\varepsilon$ is the plastic strain and $\dot{\varepsilon}$ is the strain rate. The described mechanism leads to a range of strain rates for which the strain-rate sensitivity of the flow stress is negative. In practice, this range of strain rates is unattainable by the process, and it is shown schematically in Figure 1. If the nominal strain rate is increased to the lower limit of the region of negative slope, the stress is sufficient for the strain rate to jump to a value beyond the upper limit of the unstable range. The strain will then obviously become localized to maintain the nominal strain rate.

The existence of a negative strain-rate sensitivity has recently been the subject of debate, after Korbel ${ }^{[6]}$ dismissed the idea of the existence of negative strain-rate sensitivity as an artifact, arguing that the PLC bands are localized plastic instabilities with at least three degrees of freedom that can be responsible for the stress drop after an increase in cross-head velocity. ${ }^{[7-9]}$

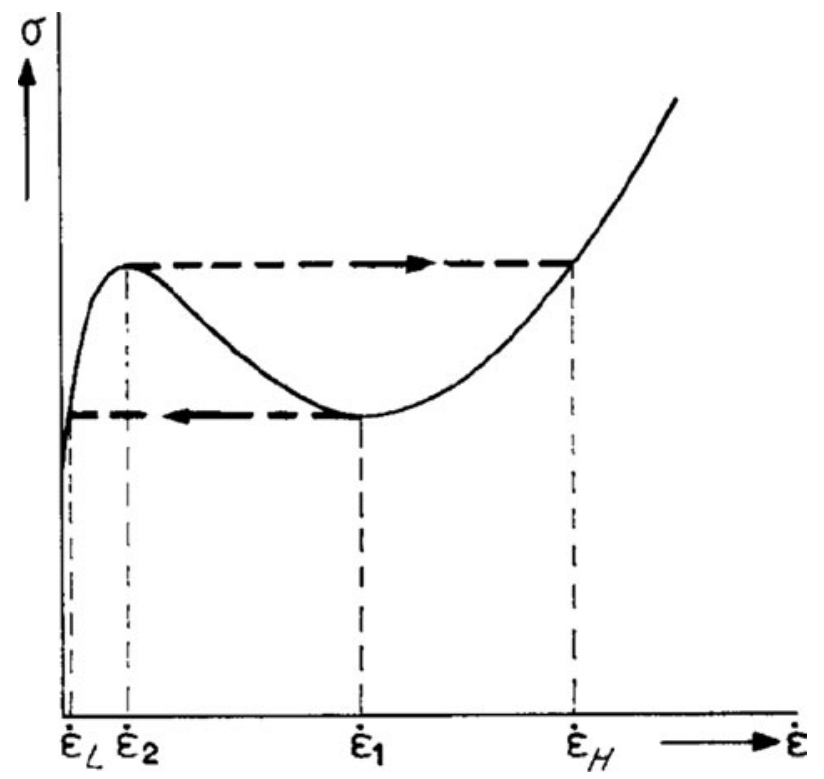

Fig. 1-Flow stress as a function of strain rate. Strain rates between $\dot{\varepsilon}_{1}$ and $\dot{\varepsilon}_{2}$ are inaccessible. ${ }^{[3]}$
Penning' ${ }^{[3]}$ theory of DSA and most subsequent work on the subject treat the strain-rate sensitivity as an instantaneous increase or decrease of the flow stress after a change in strain rate. This is the normal case for steel at room temperature, where the strain-rate sensitivity is related to thermally activated glide of dislocations.

It is widely recognized that at increased temperatures, including the range in which DSA occurs, the workhardening rate $\Theta=\frac{\partial \sigma_{\mathrm{f}}}{\partial \varepsilon}$, where $\sigma_{\mathrm{f}}$ is the flow stress, which is also a function of temperature $T$ and strain rate $\dot{\varepsilon}$. Obviously, the strain-rate influence on $\Theta$ will not cause an immediate change in flow stress when the strain rate changes: The effect will become significant on continued straining. Some authors, therefore, use the terminology "instantaneous strain-rate sensitivity" for the strain-rate sensitivity associated with thermally activated glide and "strain-rate sensitivity of work hardening" for the strain-rate sensitivity of the athermal stress. ${ }^{[10]}$ Also, "explicit" respectively "hidden" rate and temperature sensitivity is used. ${ }^{[11]}$ We will use the terminology "direct strain-rate sensitivity (DSRS)" and "indirect strain-rate sensitivity (ISRS)" for strain-rate sensitivity of the flow stress and the work-hardening rate, respectively. The difference between these two is illustrated qualitatively in Figure 2.
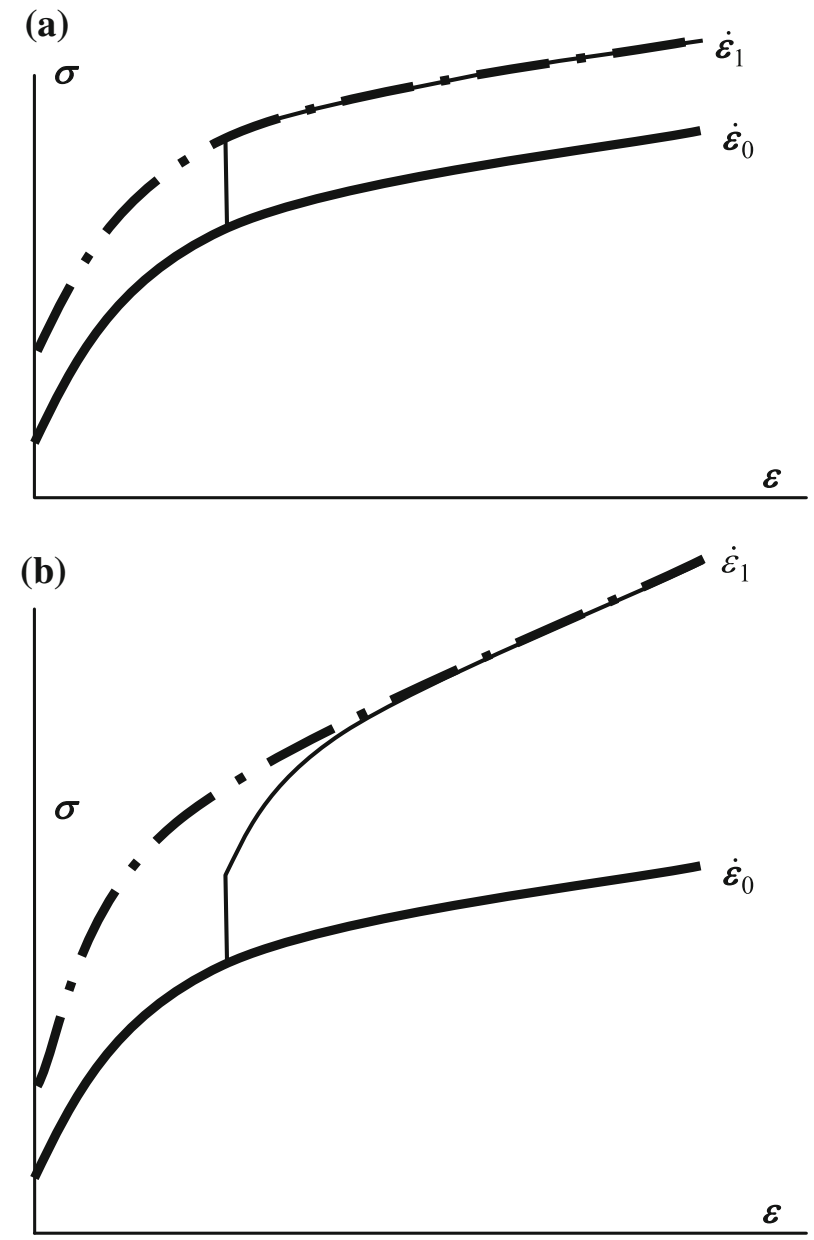

Fig. 2-Stress response to a strain-rate jump in the case of DSRS only $(a)$ and both ISRS and DSRS $(b)$. 
Figure 2(a) shows the behavior of a material exhibiting athermal work hardening and thermally activated dislocation glide. The stress response to the strain rate jump is then instantaneous. Figure 2(b) shows the response of a material exhibiting thermally activated effects on the work-hardening rate, in addition to thermally activated dislocation glide. The response then shows an instantaneous change followed by a transient in stress. Similar behavior is expected for temperature changes. In general, the work hardening of basecentered-cubic (bcc) metals, like $\alpha-\mathrm{Fe}$, is athermal at low temperatures, whereas the thermally activated dislocation glide resistance is large at those temperatures. At higher temperatures, where the glide resistance becomes negligible, the work hardening of $\alpha-\mathrm{Fe}$ is dependent on temperature and strain rate because of interactions between dislocations and diffusing point defects. For face-centered-cubic (fcc) materials like Al, the thermal effects on the work hardening dominate the thermally activated glide resistance effects at room temperature. $^{[12]}$

Physically, the existence of ISRS can be explained as follows. The contributions to the flow stress $\sigma_{\mathrm{f}}$ can be written as

$$
\sigma_{\mathrm{f}}=\sigma_{0}+\alpha G \mathbf{b} \sqrt{\rho}+\sigma^{*}
$$

where $\sigma_{0}$ is the sum of lattice resistance, solute hardening, precipitation hardening and Hall-Petch hardening. The second and third term are results of the work hardening and the contribution of thermally assisted dislocation glide $\sigma^{*}$, which is associated with shortrange interactions between dislocations or between dislocations and the crystal lattice. The parameter $\alpha$ is a crystallographic constant; $G$ is the shear modulus, which is temperature dependent; and $\mathbf{b}$ is the Burgers vector. The variable $\rho$ denotes the dislocation density, which in general increases with increasing strain $\varepsilon$. The thermal stress $\sigma^{*}$, which is the stress contribution associated with thermally activated passing of local obstacles by mobile dislocations, is only nonzero during deformation and is the cause of the direct strain-rate sensitivity. ${ }^{[13]}$

Work hardening is a contribution to the flow stress that is not thermally activated because it is caused by the long-range stresses generated by the dislocation substructure resulting from the net storage rate associated with dislocation generation and recovery processes. However, for homologous temperatures above $T h=0.2 / 0.3$, diffusing solutes and vacancies interact with the recovery mechanism, which causes the workhardening rate to become a function of strain rate and temperature. In that case, the work-hardening term in Eq. [1] becomes a function of strain rate and temperature in addition of being dependent of strain. Note the interesting feature of work hardening, which is associated with the essentially athermal process of dislocation storage, is nevertheless a function of strain rate and temperature. This is associated directly with the existence of the indirect strain-rate sensitivity, which is explained by the following.
The increase of the dislocation density during deformation follows an evolution law that can be formally be written as ${ }^{[14]}$

$$
\frac{d \rho}{d \varepsilon}=H-R(\dot{\varepsilon}, T)
$$

where $H$ is the dislocation storage rate and $R$ is the recovery rate. In the Bergström model, ${ }^{[15]} U$ is interpreted as the rate of immobilization of mobile dislocations by trapping in the dislocation substructure, and $R$ is the probability of remobilization and annihilation of immobile dislocations.

By Eqs. [1] and [2], any change caused by strain-rate changes in flow stress that is attributable to the workhardening contribution is transient in nature. The strain-rate sensitivity that is associated with work hardening is therefore indirect. The activation energy of the work-hardening term is associated with recovery because of the dislocation climb caused by vacancy diffusion, ${ }^{[16,17]}$ whereas the dislocation glide resistance is related to dislocation kink pair formation or forest dislocation cutting in the case of bcc respectively fcc metals. ${ }^{[18]}$

Many authors have found that a distinguishing feature of DSA is the increased work-hardening rate. ${ }^{[19-23]}$ The effect has been proposed as an alternative hardening mechanism to work hardening at room temperature by some investigators ${ }^{[24,25]}$ or as an increased bake-hardening effect. ${ }^{[21]}$ and Barnby ${ }^{[26]}$ reported measurements of increased dislocation densities after deformation at temperatures up to $700 \mathrm{~K}\left(427{ }^{\circ} \mathrm{C}\right)$. Static strain aging is also known to cause an increase of the work-hardening rate. ${ }^{[27,28]}$ It can therefore be concluded that strain aging and therefore the strain rate does affect the work-hardening rate. An adequate stability criterion for the occurrence of Portevin-Le Châtelier instabilities should therefore take the strainrate sensitivity of the work-hardening rate into account.

\section{STABILITY CRITERION}

Estrin and $\mathrm{Kubin}^{[4,5]}$ analyzed the stability of a strain and strain-rate sensitive material subjected to a local disturbance in plastic strain. For purposes of stability analysis, the constitutive equation describing the flow stress as a function of strain and strain rate is linearized around a working point $\sigma_{\mathrm{f}}(\varepsilon, \dot{\varepsilon})=\sigma_{\mathrm{f}}\left(\varepsilon_{0}, \dot{\varepsilon}_{0}\right)$, according to

$$
\sigma_{\mathrm{f}}(\varepsilon, \dot{\varepsilon})=\sigma_{\mathrm{f}}\left(\varepsilon_{0}, \dot{\varepsilon}_{0}\right)+\Theta\left(\varepsilon-\varepsilon_{0}\right)+S \cdot\left(\ln \left(\frac{\dot{\varepsilon}}{\dot{\varepsilon}_{0}}\right)\right)
$$

where $\Theta=\left.\frac{\partial \sigma_{\mathrm{f}}}{\partial \varepsilon}\right|_{\dot{\varepsilon}}$ is the work-hardening rate at constant strain rate, and $S=\frac{\partial \sigma_{\mathrm{f}}}{\partial \ln (\dot{\varepsilon})}$ is the strain-rate sensitivity at constant strain. The variation of stress $\delta \sigma$ caused by fluctuations $\delta \varepsilon$ in strain and $\delta \sigma$ in strain rate then reads:

$$
\delta \sigma=\Theta \delta \varepsilon+S \frac{\delta \dot{\varepsilon}}{\dot{\varepsilon}}
$$


This equation was proposed originally by Penning ${ }^{[3]}$ in a slightly different form, under the simplifying assumption that $\sigma_{\mathrm{f}}(\varepsilon, \dot{\varepsilon})$ is separable in the variables $\varepsilon$ and $\dot{\varepsilon}$.

Noting that from stress equilibrium and plastic incompressibility, it follows that

$$
\delta \sigma=\sigma \delta \varepsilon
$$

the analysis proposed in References 4 and 5 continues by introducing a disturbance in plastic strain in the mathematical form

$$
\delta \varepsilon=(\delta \varepsilon)_{0} \exp (\lambda t)
$$

where $(\delta \varepsilon)_{0}$ is the magnitude of the disturbance at $t=0$ and $\lambda$ is a growth parameter. A negative value for $\lambda$ implies that fluctuations fade with a time constant $1 / \lambda$, whereas positive values of $\lambda$ mean that the disturbance will grow into an instability. The value of $\lambda$ is a consequence of the deformation behavior, notably $\Theta, S$, and $\sigma$. Equations [4] through [6] lead to an expression for $\lambda$ in terms of the deformation characteristics given by

$$
\lambda=\frac{-\left(\Theta-\sigma_{\mathrm{f}}\right)}{S} \dot{\varepsilon}
$$

In obtaining this result, the relation $\delta \dot{\varepsilon}=\lambda(\delta \varepsilon)_{0} \exp (\lambda \varepsilon)=\lambda \delta \varepsilon$, which follows directly from Eq. [6], was used. In the case $\Theta>\sigma_{\mathrm{f}}$, where the tensile test is intrinsically stable, i.e., before necking, noting that the equivalent strain rate $\dot{\varepsilon}$ is positive and considering that $\lambda>0$ leads to instabilities, Estrin and Kubin's instability criterion reads

$$
S<0
$$

So, within this framework, negative strain-rate sensitivity is a necessary and sufficient requirement for PLC instabilities to occur. Equation [8] forms the basis of many subsequent analyses in the literature. ${ }^{[20,29-32]} \mathrm{Zbib}$ and Aifantis, ${ }^{[33]}$ working from the same assumptions as used in Reference 4 arrived at Eq. [8] by means of a nonlinear instability analysis using strain gradient plasticity.

The strain-rate sensitivity parameter $S$ in Eq. [4] can be identified as pertaining to the stress contribution caused by thermally activated dislocation glide. This is, however, not the only strain-rate dependent factor in the constitutive equation, and consequently Eq. [4] is not the total differential with respect to the strain rate.

A more comprehensive formulation takes into account also the strain-rate sensitivity of the workhardening rate (ISRS), leading to

$$
\delta \sigma=\Theta \delta \varepsilon+\varepsilon \frac{\partial \Theta}{\partial \dot{\varepsilon}} \delta \dot{\varepsilon}+\frac{S}{\dot{\varepsilon}} \delta \dot{\varepsilon}
$$

Estrin and Kubin ${ }^{[4]}$ assumed the term from the strainrate dependence of the work-hardening rate to be zero. As discussed in Section II, evidence suggests that $\frac{\partial \Theta}{\partial \dot{\varepsilon}}$ is not negligible and that the extra term in Eq. [8] can therefore have a significant effect. This leads to a revised expression for $\lambda$ derived from Eq. [9] in the same way as Eq. [7] was derived from Eq. [4], which is written as

$$
\lambda=\frac{-(\Theta-\sigma)}{\varepsilon \psi+S} \dot{\varepsilon}
$$

where $\Psi=\frac{\partial \Theta}{\partial \ln (\dot{\varepsilon})}$. An alternative criterion for PLC instability can now be formulated:

$$
\frac{(\Theta-\sigma)}{\varepsilon \Psi+S}<0
$$

For the strain range where $\Theta>\lambda$, the conclusion is that instability will occur if

$$
{ }_{\varepsilon} \Psi+S<0
$$

which is the extended stability criterion.

In the case where $\Psi$ is negative and $S$ is positive, the appearance of the strain $\varepsilon$ in Eq. [11] predicts that instability will start when a critical strain $\varepsilon_{c}$ is exceeded, which is given by

$$
\varepsilon_{c}=-\frac{S}{\psi}
$$

Equation [11] implies that $\varepsilon_{c}<0$ if $S$ and $\Psi$ have the same sign, in which case instabilities will occur from the start of the deformation process. For positive values of $S$ and negative $\Psi$, sudden stress drops are not expected to occur because those must be associated with a negative value of $S$, which describes direct response on a strain-rate jump. The indirect strain-rate sensitivity $\Psi$, which is associated with work hardening, can only cause transient stress changes. Probably, the deformation bands will propagate steadily in this case, with gentle undulations of the flow-stress curve. Within the scope of this article, we will not consider subsequent analysis and modeling of the dynamics of the PLC instabilities as in References 29-32.

\section{DISCUSSION}

The conventional explanation of the PLC effect is based on the concept of negative direct strain-rate sensitivity. The published theoretical models focus on aging effects acting on the mobile dislocations and address only possible changes of the thermal stress that are associated with thermally activated glide of mobile dislocations. Although recent publications ${ }^{[34,35]}$ criticize the proposed physical mechanisms in previous publications, the models proposed by those authors are restricted to a description of DSA effects pertaining to mobile dislocations. The assumed mechanism in these theories is solute drag on mobile dislocations by interstitial solutes that have segregated to the dislocations forming a Cottrell atmosphere. ${ }^{[36,37]}$

Experimental evidence has to be treated with caution, first because during PLC instabilities, the local strain rate inside the deformation bands will be higher than the 
nominal strain rate, and second because the range of strain rates where DSRS is negative is experimentally inaccessible (Figure 1). Practically, the feature of the theory pertaining to negative values of DSRS is particularly awkward, as it precludes a meaningful confrontation of the theory with the experiment. In contrast, the work-hardening rate and its strain-rate sensitivity are observable quantities, and it is worthwhile to analyze experimental data and relate observed strain-rate sensitivities to the occurrence of the PLC -instabilities.

Not much attention has been paid in literature to the strain-rate sensitivity of the work-hardening rate associated with DSA and to the problem whether it exhibits negative values. Keh et al. ${ }^{[38]}$ reported "an inverse strain rate dependence of the flow stresses... [that] shifted to higher strain rates at still higher temperatures." Kocks et al. ${ }^{[39]}$ observed that, "It is evident that the lower strain rate causes more hardening." This influence of DSA on the work-hardening rate is not surprising, as obviously the aging time, which can be defined as the time available for a sufficient number of solutes to diffuse to a dislocation segment to produce locking, is greater for immobile dislocations than for mobile dislocations. The aging time can be identified with the resting time of immobile dislocations between a storage event and a remobilization/annihilation event in the Bergström model. ${ }^{[40]}$ The immobile dislocations therefore have a greater probability than mobile dislocations to acquire a Cottrell atmosphere and be locked or pinned by it. This will in turn decrease the (dynamic) recovery rate, which leads to an increased work-hardening rate. At high strain rates, the ratio of the locking rate to the rate of dislocation storage will decrease, which explains the negative value of the work-hardening rate.

Referring to aging of mobile dislocations only, Soare and Curtin" ${ }^{[35]}$ observed that "bulk diffusion of $\mathrm{Mg}$ in $\mathrm{Al}$ at room temperature is far too slow, by a factor of $10^{6}$, to account for the dynamic strain-aging effects observed" and that "pipe diffusion is also inadequate for accelerating the accumulation of solutes in the dislocation core." This observation suggests that the mechanism of pinning of mobile dislocations, which is associated with DSRS, is insufficient to explain Portevin-Le Châtelier instabilities. Therefore, in addition to negative direct strain-rate sensitivity, an additional effect is influencing the mechanical behavior. We show using published experimental data that ISRS is negative in the range of temperatures and strain rates where PortevinLe Châtelier instabilities occur.

Karimi Taheri et al. ${ }^{[41]}$ published a series of tensile curves of low carbon steel that show clearly the anomaly in hardening rate and its dependence of temperature and strain rate, which are reproduced in Figure 3. Additionally they published the flow stress at several constant strain values as a function of temperature for a wide range of strain rates. Because of the completeness of these data in combination with the prominent anomalous hardening peak, we chose this publication as a basis for the subsequent quantitative analysis. Similar but less comprehensive data or results from experiments on alloys showing a less prominent effect can be found in References 21, 22, 24, 26, 38 and 42 .
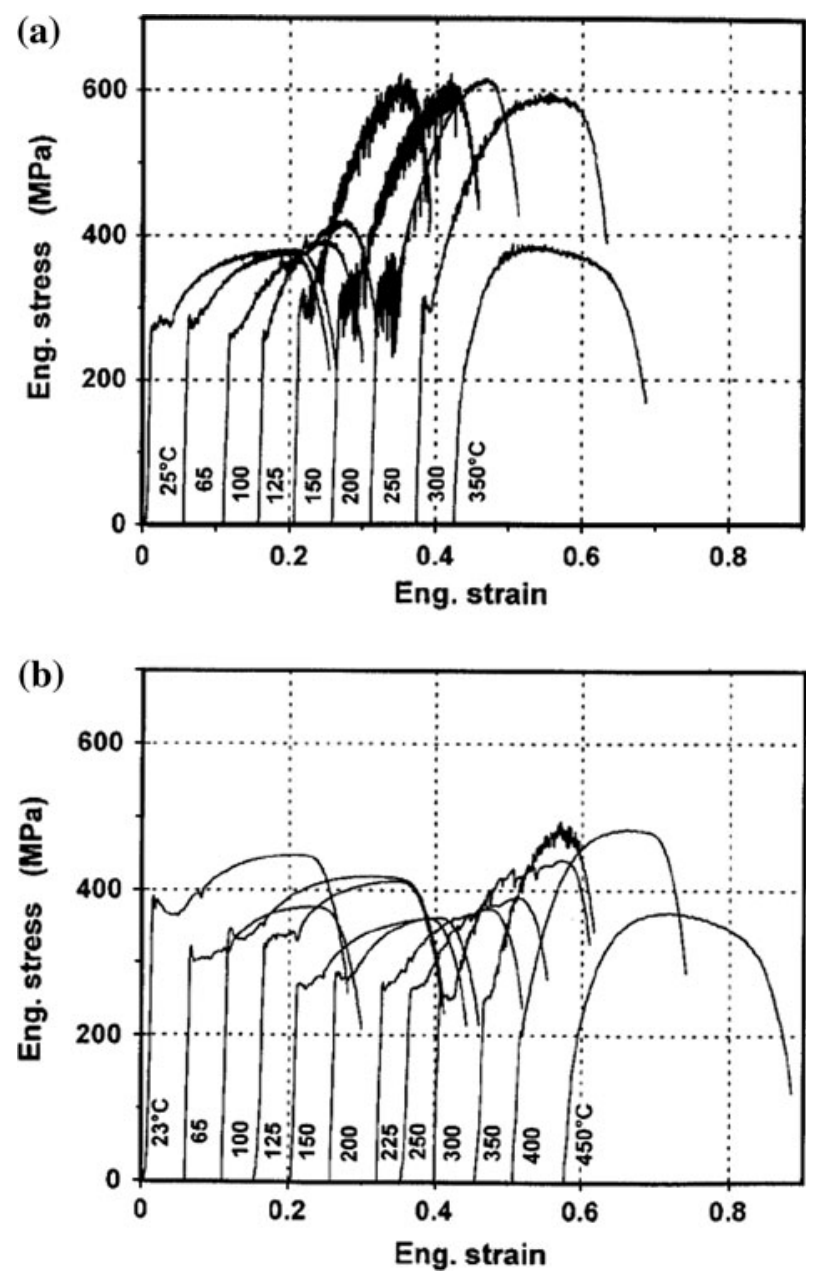

Fig. 3-Stress vs strain curves for specimens tensile tested at various temperatures and a strain rate of: $(a) \dot{\varepsilon}=10^{-4}\left(\mathrm{~s}^{-1}\right)$ and $(b) \dot{\varepsilon}=10^{-1}$ $\left(\mathrm{s}^{-1}\right) \cdot{ }^{[41]}$

A comparison of the mechanical behavior for $T \leq 373 \mathrm{~K}\left(100^{\circ} \mathrm{C}\right)$ for low strain rate (Figure $3(\mathrm{a})$ ) with that high strain rate (Figure 3(b)) shows that the work-hardening rates at both strain rates do not differ significantly (the curves have the same shape). Therefore, the strain-rate sensitivity in these experiments should be classified as direct, and it is positive for this temperature range.

At temperatures above $373 \mathrm{~K}\left(100{ }^{\circ} \mathrm{C}\right)$ the shape of the hardening curve changes conspicuously, and the work-hardening behavior becomes sensitive to the strain rate. This can be classified as indirect strain-rate sensitivity. Because the work hardening is clearly stronger in Figure 2(a) than in Figure 2(b), ISRS is negative for $373 \mathrm{~K}\left(100^{\circ} \mathrm{C}\right)<T<623 \mathrm{~K}\left(350^{\circ} \mathrm{C}\right)$. The flowstress curves are jerky in this range, so that we may conclude that both the PLC instabilities and negative ISRS are caused by DSA.

At temperatures above $623 \mathrm{~K}\left(350^{\circ} \mathrm{C}\right)$, the DSA effect decreases, and it will eventually be superseded by dynamic recovery because of which ISRS becomes positive.

From the flow stress vs temperature/strain rate data, ${ }^{[41]}$ an estimate of the strain-rate sensitivity of the 


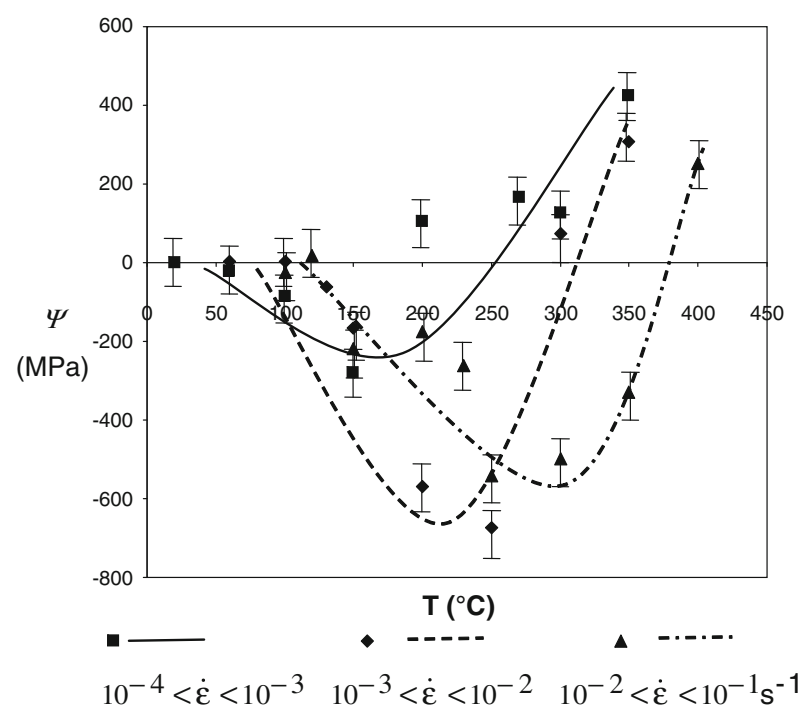

Fig. 4 - Strain-rate sensitivity $\Psi$ of the work-hardening rate. Computed from Ref. 41. The errors are estimated from PLC fluctuations in Fig. 3.

work-hardening rate $\Psi=\frac{\partial \Theta}{\partial \ln (\dot{\varepsilon})}$ as a function of temperature for different strain-rate intervals was made (Figure 4). From these results, it is observed that ISRS becomes negative in the temperature ranges where instabilities occur. Figure 3 shows that the instabilities vanish at temperatures above $523 \mathrm{~K}$ and $623 \mathrm{~K}\left(250^{\circ} \mathrm{C}\right.$ and $350^{\circ} \mathrm{C}$ ) for strain rates of $10^{-4}$ seconds $^{-1}$ and $10^{-1}$ seconds ${ }^{-1}$, respectively, which is in agreement with the crossover of $\Psi$ to positive values shown in Figure 4. The position of the minimum in $\Psi$ shifts to higher temperatures if the strain rate is increased. The magnitude of $\left|\frac{\partial \Theta}{\partial \ln (\bar{\varepsilon})}\right|$ is $400-700 \mathrm{MPa}$. It must be stressed that these are nominal values. Because of the strain localization during the PLC instabilities, the local strain rate will be larger than the nominal strain rate. The physically correct value of $\Psi$, which has to be computed from local strain rates, will differ from the nominal value in cases where PLC instability occurs.

The values of $S$ for low carbon steels used in early work on negative DSRS values are in the range $S=1-$ $2 \mathrm{MPa}{ }^{[43]}$ The term $\Psi$ in Eq. [11] will therefore exceed $S$ at small values of strain (less than 0.5 pct with $\left|\frac{\partial \Theta}{\partial \ln ((\dot{\varepsilon})}\right|$ being $400-700 \mathrm{MPa}$ ) and cannot be neglected in the analysis of PLC instabilities and in models that aim to predict the dynamics of PLC band nucleation and propagation.

The remaining question to be answered lies in the physical explanation of the negative strain-rate sensitivity of the work-hardening rate. In contrast to the state of affairs regarding modeling the dynamic aspects of DSA, models of the increased work-hardening rate during DSA are few in number. The Bergström model ${ }^{[15,40]}$ has been developed to describe the increase of work-hardening rate in the case of $\mathrm{Fe}$ containing free interstitial solutes. ${ }^{[4]}$ The Bergström model is mathematically equivalent to the Kocks-Mecking model, but it differs in the interpretation of the recovery term. In the
Bergström theory, remobilization of immobile dislocations is a secondary source of mobile dislocations in addition to dislocation creation. At higher temperatures, the remobilization process is enhanced by dislocation climb because of vacancy diffusion, which lowers the work-hardening rate. At increasing strain rates, this effect decreases so that the strain-rate sensitivity of the work-hardening rate (ISRS) caused by this effect is positive. The remobilization concept is similar to that of dynamic recovery, albeit that in the prevailing KocksMecking interpretation recovery is viewed as an annihilation mechanism. Neither the Bergström nor the Kocks-Mecking model offer a viable explanation for the increased work-hardening rate for conditions where DSA occurs.

In the extended Bergström model, ${ }^{[44]}$ the increased rate of work hardening is explained by locking of immobile dislocations by diffusing solutes, which renders them ineffective for remobilization. This interaction leads to an opposite effect as in the case of dislocationvacancy interaction: The strain-rate sensitivity of the work-hardening rate will be negative. Consequently, the creation rate of mobile dislocations will have to increase to fulfill the requirement that the mobile dislocation density remain constant. The model recognizes locked and free immobile dislocations with respect to their ability to remobilize. Formally, a locking rate term is added in this model to the dislocation evolution Eq. [2], which then reads

$$
\frac{d \rho}{d \varepsilon}=H-R(\dot{\varepsilon}, T)+L(\dot{\varepsilon}, T)
$$

A more detailed description of this model can be found in Reference 44. The model was used to describe experimental data in the stable austenite temperature range, which led to the conclusion that DSA occurs in austenite as well as in ferrite. The model predicts that ISRS is negative for temperatures where DSA occurs. This can be understood as follows: DSA increases the work-hardening rate by locking/ pinning immobile dislocations. At high deformation rates, the remobilization rate increases, and the probability of locking will decrease. The increase in work-hardening rate as a result of DSA will then be lower than for a low strain rate. In other words, during DSA, the hardening rate decreases with increasing strain rate.

\section{CONCLUSIONS}

In this article, an extended criterion for the onset of Portevin-Le Châtelier instabilities is presented. The proposed criterion is an extension of the Kubin-Estrin criterion by including the indirect strain-rate sensitivity. The criterion indicates that plastic deformation will become unstable at a critical strain if the direct strainrate sensitivity or the indirect strain-rate sensitivity (or both, in which case the critical strain is zero) becomes negative. 
Experimental evidence is presented that shows ISRS to be negative in the range where DSA/PLC instabilities occur. An analysis of literature data showed the contribution of ISRS to stress variations to be of the same order of magnitude or even larger than the DSRS contribution. Consequently, the strain-rate sensitivity of the work-hardening rate should be taken into account in dynamical models of PLC instabilities.

The occurrence of negative ISRS values can be explained by the extended Bergström model. If this model is used to describe a comprehensive set of tensile data, it can be employed in conjunction with the derived stability criterion to map the strain rate/temperature regime where instabilities will occur.

\section{OPEN ACCESS}

This article is distributed under the terms of the Creative Commons Attribution Noncommercial License which permits any noncommercial use, distribution, and reproduction in any medium, provided the original author(s) and source are credited.

\section{REFERENCES}

1. R.A. Mulford and U.F. Kocks: Acta Metall., 1979, vol. 27, pp. 1125-34.

2. C. Schwink and A. Nortmann: Mater. Sci. Eng. A, 1997, vols. 234 236, pp. 1-7.

3. P. Penning: Acta Metall., 1972, vol. 20, pp. 1169-75.

4. Y. Estrin and L.P. Kubin: Mater. Sci. Eng. A, 1991, vol. 137, pp. $125-34$.

5. L.P. Kubin and Y. Estrin: Acta Metall., 1985, vol. 33 (3), pp. $397-$ 407.

6. A. Korbel and H. Dybiec: Acta Metall., 1980, vol. 29, pp. 89-93.

7. H. Dybiec: Scripta Metall., 1988, vol. 22, pp. 595-99.

8. L.P. Kubin and Y. Estrin: Scripta Metall., 1989, vol. 23, pp. 81517

9. H. Dybiec: Scripta Metall., 1989, vol. 23, pp. 1997-2000

10. J.R. Klepaczko and C.Y. Chiem: J. Mech. Phys. Solids, 1986, vol. 34 (1), pp. 29-54

11. K. Domkin: Masters Thesis, Luleå University of Technology, Luleå, Sweden, 2005.

12. F.J. Zerilli: Metall. Mater. Trans. A, 2004, vol. 35A, pp. 2547-55.

13. U.F. Kocks and H. Mecking: Progr. Mater. Sci., 2003, vol. 48, pp. 171-273.

14. H. Mecking and U.F. Kocks: Acta Metall., 1981, vol. 29, pp. 1865-75.
15. Y. Bergström: Mater. Sci. Eng., 1969/70, vol. 5, pp. 193-200.

16. E. Nes, K. Marthinsena, and Y. Brechet: Scripta Mater., 2002, vol. 47, pp. 607-11.

17. H.P. Stüwe: Acta Metall., 1965, vol. 13, pp. 1337-42.

18. C.Y. Gaoa and L.C. Zhang: Mater. Sci. Eng. A, 2010, vol. 527, pp. 3138-43.

19. C.G. Schmid and A.K. Miller: Acta Metall., 1982, vol. 30, pp. 615-35.

20. J. Cheng and S. Nemat-Nasser: Acta Mater., 2000, vol. 48, pp. 3131-44

21. K. Dehghani and J.J. Jonas: Metall. Mater. Trans. A, 2000, vol. $31 \mathrm{~A}$, pp. $1375-84$.

22. K. Dehghani: Masters Thesis, McGill University, Montreal, Canada, 1999.

23. G.A. Henshall and A.K. Miller: Acta Metall., 1989, vol. 37 (10), pp. 2693-2704

24. D.J. Lloyd and K. Tangri: Mater. Sci. Eng., 1972, vol. 10, pp. 7580.

25. W.A. Spitzig and R.J. Sober: Mater. Sci. Eng., 1975, vol. 20, pp. 179-84

26. B.J. Brindley and J.T. Barnby: Acta Metall., 1966, vol. 14, pp. $1765-80$

27. D.V. Wilson and B. Russell: Acta Metall., 1960, vol. 8, pp. 36-45.

28. W.C. Leslie: The Physical Metallurgy of Steels, McGraw-Hill, New York, NY, 1981.

29. S. Rajesh and G. Ananthakrishna: Phys. Rev. E, 2000, vol. 61 (4), pp. 3664-74.

30. C. Fressengeas, A.J. Beaudoin, M. Lebyodkin, L.P. Kubin, and Y. Estrin: Mater. Sci. Eng. A, 2005, vols. 400-401, pp. 226-30.

31. G. Ananthakrishna and M.S. Bharathi: Phys. Rev. E, 2004, vol. 70, pp. 026111-1-15

32. H. Ait-Amokhtara, C. Fressengeas, and S. Boudrahema: Mater. Sci. Eng. A, 2008, vol. 488, pp. 540-46.

33. H.M. Zbib and E.C. Aifantis: Scripta Metall., 1988, vol. 22, pp. 1331-36.

34. P. Hähner: Mater. Sci. Eng. A, 1996, vol. 207, pp. 208-15.

35. M.A. Soare and W.A. Curtin: Acta Mater., 2008, vol. 56, pp. 4046-61.

36. A.H. Cottrell and B.A. Bilby: Proc. Phys. Soc. LXII, 1948, vol. IA, pp. 49-62.

37. J.Z. Zhao, A.K. De, and B.C. De Cooman: Metall. Mater. Trans. A, 2001, vol. 32A, pp. 417-23.

38. A.S. Keh, Y. Nakada, and W.C. Leslie: Dislocation Dynamics, Ed. A.R. Rosenfield, McGraw-Hill, New York, NY, 1968, pp. 381408

39. U.F. Kocks, R.E. Cook, and R.A. Mulford: Acta Metall., 1985, vol. 33 (4), pp. 623-38.

40. Y. Bergström: Rev. Powder Met. Phys. Cer., 1983, pp. 79-265.

41. A. Karimi Taheri, T.M. Maccagno, and J.J. Jonas: ISIJ Int., 1995, vol. 35 (12), pp. 1532-40.

42. A. Gilat and X. Wu: Int. J. Plast., 1997, vol. 13 (6-7), pp. 61132.

43. P.G. McCormick: Acta Metall., 1988, vol. 36 (12), pp. 3061-67.

44. P. van Liempt, M. Onink, and A. Bodin: Adv. Eng. Mat., 2002, vol. 4, pp. 225-32. 